Virginia Commonwealth University

vCU Scholars Compass

2011

\title{
Extremely high absolute internal quantum efficiency of photoluminescence in co-doped GaN:Zn,Si
}

Michael A. Reshchikov

Virginia Commonwealth University,mreshchi@vcu.edu

A. G. Willyard

Virginia Commonwealth University

A. Behrends

Institute of Semiconductor Technology

A. Bakin

Institute of Semiconductor Technology

A. Waag

Institute of Semiconductor Technology

Follow this and additional works at: http://scholarscompass.vcu.edu/phys_pubs

Part of the Physics Commons

Reshchikov, M.A., Willyard, A.G., Behrends, A., et al. Extremely high absolute internal quantum efficiency of photoluminescence in co-doped GaN:Zn,Si. Applied Physics Letters, 99, 171110 (2011). Copyright (C) 2011 AIP Publishing LLC.

\section{Downloaded from}

http://scholarscompass.vcu.edu/phys_pubs/27

This Article is brought to you for free and open access by the Dept. of Physics at VCU Scholars Compass. It has been accepted for inclusion in Physics Publications by an authorized administrator of VCU Scholars Compass. For more information, please contact libcompass@vcu.edu. 


\title{
Extremely high absolute internal quantum efficiency of photoluminescence in co-doped GaN:Zn,Si
}

\author{
M. A. Reshchikov, ${ }^{1, a)}$ A. G. Willyard, ${ }^{1}$ A. Behrends, ${ }^{2}$ A. Bakin, ${ }^{2}$ and A. Waag ${ }^{2}$ \\ ${ }^{1}$ Physics Department, Virginia Commonwealth University, Richmond, Virginia 23284, USA \\ ${ }^{2}$ Institute of Semiconductor Technology, Braunschweig University of Technology, Braunschweig, Germany
}

(Received 27 July 2011; accepted 3 October 2011; published online 27 October 2011)

\begin{abstract}
We report on the fabrication of $\mathrm{GaN}$ co-doped with silicon and zinc by metalorganic vapor phase epitaxy and a detailed study of photoluminescence in this material. We observe an exceptionally high absolute internal quantum efficiency of blue photoluminescence in GaN:Zn,Si. The value of $0.93 \pm 0.04$ has been obtained from several approaches based on rate equations. (C) 2011 American Institute of Physics. [doi:10.1063/1.3655678]
\end{abstract}

Co-doping GaN by Si and Zn leads to bright emission at a photon energy of about $2.9 \mathrm{eV} .{ }^{1}$ Since this is in the blue spectral range, such a co-doping has been used in early LED structures for creating blue light emission. ${ }^{2}$ However, after the "invention" of the InGaN quantum well, the Zn-related emission has no longer been used in LEDs. New interest in this material could arise from the field of single photon emitters, and high internal quantum efficiency (IQE) is a prerequisite in this case.

Whereas the external quantum efficiency can be determined by merely measuring the number of photons emitted by the LED chip, the determination of the IQE of electroluminescence or photoluminescence (PL) is much more indirect. Often, the ratio of room-temperature and lowtemperature PL intensity is used as a rough estimate of the relative IQE. There is no consensus in literature on how to determine the absolute IQE of PL in GaN. High values of the absolute IQE of PL in GaN or InGaN were reported, in the range of $30 \%-70 \%{ }^{3-6}$ However, assumptions for the method used in these publications to estimate the absolute IQE require better justification.

In this work, we report on extremely high absolute IQE of PL in degenerate $n$-type $\mathrm{GaN}$ co-doped with $\mathrm{Zn}$ and Si. The IQE was determined from analysis of the temperature and excitation intensity dependences of PL. The absolute IQE of the blue luminescence (BL) band in GaN:Zn,Si exceeds $90 \%$.

Samples for this work were grown by metalorganic vapor phase epitaxy (MOVPE) on $c$-plane sapphire substrates. After growing $2 \mu \mathrm{m}$-thick undoped $\mathrm{GaN}$, a $0.4 \mu \mathrm{m}$ thick AlGaN barrier was grown, followed by a $0.2 \mu$ m-thick GaN:Zn,Si layer and capped with a $40 \mathrm{~nm}$-thick AlGaN barrier. The concentration of free electrons in the GaN:Zn,Si layer has been estimated as $3 \times 10^{18} \mathrm{~cm}^{-3}$ from electrochemical C-V (ECV) profiling measurements and $5 \times 10^{18} \mathrm{~cm}^{-3}$ from time-resolved PL experiments. The PL lifetime of the BL band $\left(\tau_{1}=0.5 \mu \mathrm{s}\right)$ was independent of temperature at $T<250 \mathrm{~K}$, i.e., the layer is degenerate. Steady-state PL was excited with a $50 \mathrm{~mW} \mathrm{He-Cd} \mathrm{laser.} \mathrm{Other} \mathrm{details} \mathrm{of} \mathrm{the} \mathrm{ex-}$ perimental set-up as well as detailed information on the method of estimating the IQE of PL can be found in Ref. 7.

A PL spectrum from GaN:Zn,Si at three representative temperatures is shown in Fig. 1. It includes a strong BL band

\footnotetext{
${ }^{a}$ Electronic mail: mreshchi@vcu.edu.
}

with a maximum at $2.9 \mathrm{eV}$, a weak exciton peak at higher photon energies, and a broad defect-related band with a maximum at about $2.1 \mathrm{eV}$. The latter will be called here the yellow luminescence (YL) band, although its identity and relation to the YL bands reported in literature ${ }^{1}$ were not established. The BL band is attributed to transitions from the conduction band (or shallow donor at very low temperature) to the $\mathrm{Zn}_{\mathrm{Ga}}$ acceptor. ${ }^{1,7}$ The shape of this band at different temperatures is well known, ${ }^{1,7}$ and this information was used to deconvolute the bands before finding their integrated intensities.

Figure 2 shows the temperature dependence of the integrated PL intensity for three PL bands in relative units (right scale). The BL band quenches at $T>250 \mathrm{~K}$ with the activation energy of about $300 \mathrm{meV}$. The characteristic temperature of the quenching $T_{0}$ can be defined as the point where the low-temperature and high-temperature parts of the dependence cross when extrapolated as straight lines in the Arrhenius plot (see Fig. 2). This value in the studied samples is unusually high $\left(T_{0}=300 \mathrm{~K}\right)$ as compared to the values reported earlier $\left(T_{0}=200-220 \mathrm{~K}\right) .^{7,8}$ The exciton and YL band intensities slowly decrease with increasing temperature from 15 to $250 \mathrm{~K}$ (not shown for $T<100 \mathrm{~K}$ ). However, simultaneously with the quenching of the BL band, namely, at $T=250-350 \mathrm{~K}$, they both exhibit a sharp rise by an

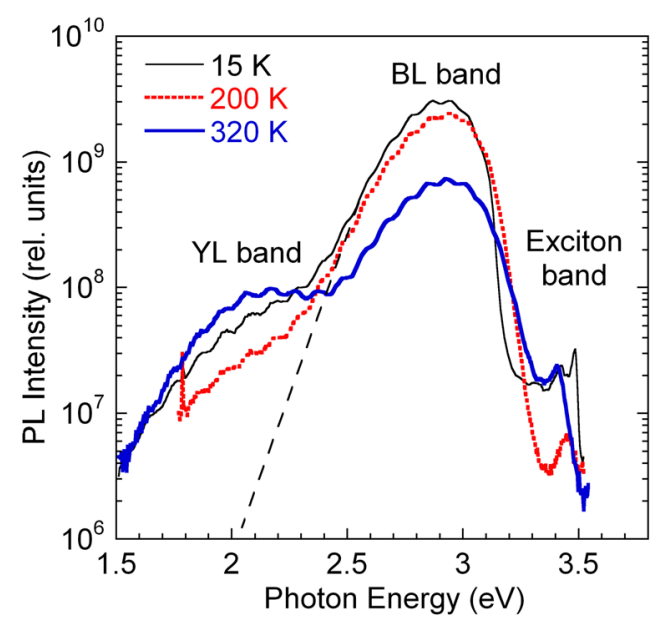

FIG. 1. (Color online) PL spectra at three representative temperatures. $P_{\text {exc }}=0.0015 \mathrm{~W} / \mathrm{cm}^{2}$. Weak oscillations are due to interference effect. Black dashed line shows the low-energy tail of the BL band in a Zn-doped GaN sample where no YL band was observed. 


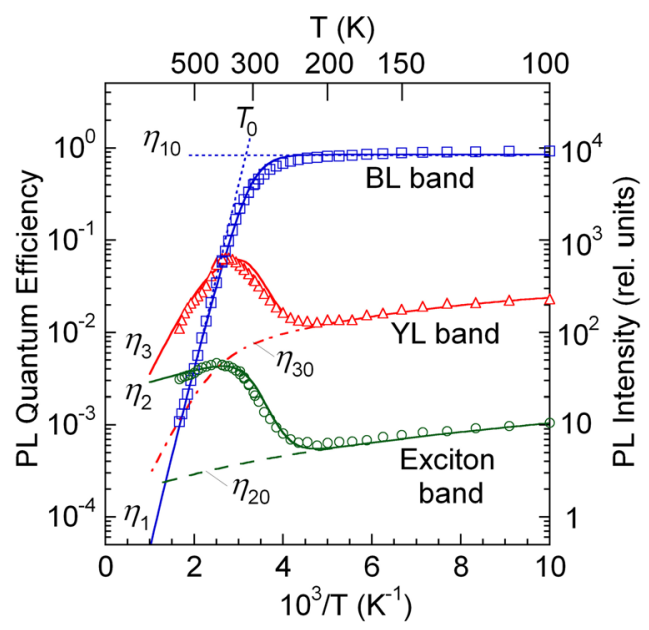

FIG. 2. (Color online) Temperature dependence of the absolute internal quantum efficiency of PL in GaN:Zn,Si at $P_{e x c}=0.0015 \mathrm{~W} / \mathrm{cm}^{2}$. Points are the experimental data. Solid curve for the BL band shows $\eta_{1}$ calculated by using Eq. (1) with the following parameters: $\eta_{10}=0.97, g=2, C_{p A}=10^{6} \mathrm{~cm}^{3} / \mathrm{s}$, $\tau_{1}=0.5 \mu \mathrm{s}$, and $E_{A}=300 \mathrm{meV}$. Dotted lines are extrapolations of the lowtemperature and high-temperature parts of the $\eta_{1}(T)$ dependence with crossing at $T_{0}$. Dashed and dash-dotted lines show $\eta_{20}(T)$ and $\eta_{30}(T)$ calculated by using Eq. (2) with the following parameters: $\eta_{20}(0)=3.7 \times 10^{-3}, C_{1}=10$, $C_{2}=13, E_{1}=12, E_{2}=50 \mathrm{meV}$ (for the exciton band), $\eta_{30}(0)=0.03$, $C_{1}=8, C_{2}=10^{3}, E_{1}=30$, and $E_{2}=200 \mathrm{meV}$ (for the YL band). $G=2.4 \times 10^{20} \mathrm{~cm}^{-3} \mathrm{~s}^{-1}$ and $\beta=4.2 \times 10^{-17} \mathrm{~cm} \mathrm{~s}$ for all the bands. Solid lines for the exciton and YL bands show $\eta_{2}(T)$ and $\eta_{3}(T)$ calculated by using Eq. (4) with $\eta_{10}=0.92$ and $\eta_{10}=0.93$, respectively.

order of magnitude and then decrease again at higher temperatures. Such a behavior clearly indicates a very high IQE of the BL band. ${ }^{8}$ Below we will explain the results and describe how the absolute IQE for the PL bands (shown on the left scale of Fig. 2) was determined.

With increasing temperature from 15 to $250 \mathrm{~K}$, the BL band intensity remained nearly constant, in agreement with the model for the BL band in $n$-type GaN: $\mathrm{Zn}$ developed in Refs. 7 and 8. The temperature dependence of the BL band IQE $\eta_{1}(T)$ in $n$-type GaN can be described with a formula ${ }^{7,8}$

$$
\eta_{1}(T)=\frac{\eta_{10}}{1+\left(1-\eta_{10}\right) \tau_{1} Q_{1}},
$$

where $\eta_{10}$ is the IQE of the BL band at low temperatures $(T<250 \mathrm{~K}), \tau_{1}$ is the BL band lifetime determined from timeresolved PL measurements, $Q_{1}$ is the hole-emission coefficient $\left(Q_{1}=C_{p A} g^{-1} N_{v} e^{-E_{A} / k T}\right), C_{p A}$ is the hole-capture coefficient for the Zn-related BL band in $\mathrm{GaN}\left(C_{p A}=10^{-6} \mathrm{~cm}^{3} / \mathrm{s}\right),{ }^{1}$ $N_{v}$ is the density of states in the valence band, $g$ is the degeneracy factor of the acceptor level, $E_{A}$ is the ionization energy of the $\mathrm{Zn}_{\mathrm{Ga}}$ acceptor $(0.40 \mathrm{eV}$ at $15 \mathrm{~K}$ and decreases to $0.35 \mathrm{eV}$ at $T \approx 200 \mathrm{~K}),{ }^{1}$ and $k$ is Boltzmann's constant. According to a phenomenological model for an $n$-type semiconductor, ${ }^{7,8}$ the thermal quenching of the strongest PL band results in simultaneous increase of recombination rate (or PL intensity) via all other recombination channels.

There are four main recombination channels in $\mathrm{GaN}: \mathrm{Zn}, \mathrm{Si}$, each with absolute IQE $\eta_{i}$. These include the BL band with $\eta_{1}$ defined with Eq. (1), exciton or near-bandedge emission with $\eta_{2}=B n p / G$, the YL band with $\eta_{3}=C_{p 3} N_{3} p / G$, and recombination via nonradiative centers with $\eta_{4}=C_{p 4} N_{4} p / G$, where $G$ is the electron-hole generation rate, $n$ and $p$ are concentrations of free electrons and holes, respectively, $B$ is the exciton or near-bandedge recombination rate coefficient, and $C_{p i}$ and $N_{i}$ are hole-capture coefficients and concentrations of different defects participating in carrier recombination. The PL intensity integrated over a spectral region of a particular PL band, $I_{i}^{P L}$, is proportional to the absolute IQE of this PL band, and it can be expressed as $I_{i}^{P L}=\beta \eta_{i} G$, where $\beta$ is a constant determined by the total collection efficiency of luminescence (same for all channels and independent of temperature).

Next, we define $\eta_{i 0}$ and $I_{i 0}^{P L}\left(I_{i 0}^{P L}=\beta \eta_{i 0} G\right)$ as the temperature-dependent absolute IQE and corresponding integrated PL intensity for the $i$ th channel $(i \neq 1)$ which would be observed if the BL band did not quench at all, as opposed to the values $\eta_{i}$ and $I_{i}^{P L}$ which include the quenching. In practice, the temperature dependence of $I_{i 0}^{P L}$ can be found by modeling the experimentally found $I_{i}^{P L}(T)$ dependence at temperatures below the quenching of the BL band with the following expression:

$$
I_{i 0}^{P L}(T)=\frac{I_{i 0}^{P L}(0)}{1+C_{1} e^{-E_{1} / k T}+C_{2} e^{-E_{2} / k T}}
$$

and extrapolating this dependence into higher temperatures. Here, $I_{i 0}^{P L}(0)=\beta \eta_{i 0}(0) G$ is the integrated PL intensity in the low-temperature limit and $C_{1}, C_{2}, E_{1}$, and $E_{2}$ are fitting parameters that determine positions and slopes of lines in the Arrhenius plot. The $I_{i 0}^{P L}(T)$ [or $\left.\eta_{i 0}(T)\right]$ dependences for the exciton $(i=2)$ and YL $(i=3)$ bands are shown in Fig. 2 with dashed and dash-dotted curves, respectively.

Since $\sum_{i=1}^{4} \eta_{i}=1$ and each term $\eta_{i}$ with $i>1$ contains the hole concentration $p$ as a factor, we can express $p$ as

$$
p=\frac{G\left(1-\eta_{1}\right)}{B n+C_{p 3} N_{3}+C_{p 4} N_{4}}
$$

and substitute this into expressions for the IQE of any channel with $i>1$. In the region of substantial thermal quenching of the BL band ( $T \approx 250-350 \mathrm{~K})$, intensities of other PL bands increase because $\eta_{1}$ in Eq. (3) decreases. If the BL band did not quench, concentration of holes would be described by the same Eq. (3) with $\eta_{1}=\eta_{10}$. Thus, by dividing $\eta_{i}$ on $\eta_{i 0}$ and cancelling out the terms that have the same temperature dependence, we can express the relative change of the integrated PL intensity $I_{i}^{P L} / I_{i 0}^{P L}$ due to the quenching of the BL band as

$$
\frac{I_{i}^{P L}}{I_{i 0}^{P L}}=\frac{\eta_{i}}{\eta_{i 0}}=\frac{1-\eta_{1}}{1-\eta_{10}} .
$$

Note that the $I_{i}^{P L} / I_{i 0}^{P L}$ ratios and their temperature dependence are identical for all recombination channels with $i>1$. Recalling that $\eta_{1}=\left(I_{1}^{P L} / I_{10}^{P L}\right) \eta_{10}$, we see that $\eta_{10}$ is the only fitting parameter in the fit of the experimentally found $I_{i}^{P L}(T)$ dependences with Eq. (4) for channels with $i>1$, provided that we have the $I_{1}^{P L}(T) / I_{10}^{P L}(T)$ dependence and simulated the $I_{i 0}^{P L}(T)(i>1)$ dependences.

The quenching of the BL band begins at much higher temperatures $\left(T_{0}=300 \mathrm{~K}\right)$ than it is commonly observed $(200-220 \mathrm{~K}) .^{1,8}$ This can be explained by the very small PL lifetime (which is due to a high concentration of free 


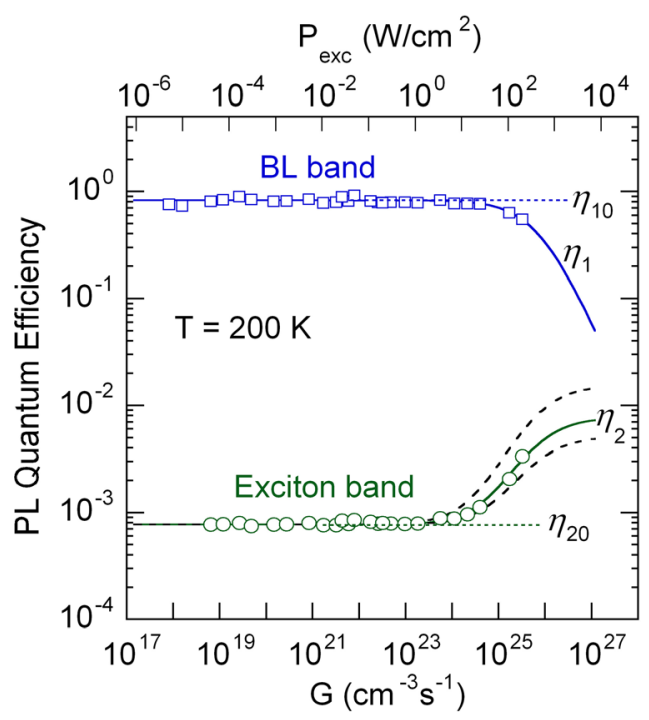

FIG. 3. (Color online) Dependence of PL quantum efficiency on excitation intensity for the BL band and exciton band at $T=200 \mathrm{~K}$. $G=1.6 \times 10^{23} \times P_{\text {exc }}$. Solid line for the exciton band shows $\eta_{2}(G)$ calculated by using Eq. (4) with $\eta_{10}=0.90$. Dashed lines show $\eta_{2}(G)$ with $\eta_{10}=0.95$ (upper curve) and $\eta_{10}=0.85$ (lower curve).

electrons) and exceptionally high IQE of the BL band $\left[\eta_{10}=0.97\right.$ from the fit of the experimental data for the BL band with Eq. (1)]. The best fits of the $I_{i}^{P L}(T)$ dependences with Eq. (4) for the exciton and YL bands are shown in Fig. 2 with solid curves. We obtained $\eta_{10}=0.92$ and $\eta_{10}=0.93$ by fitting the $I_{i}^{P L}(T)$ dependences for the exciton and YL bands, respectively. Very close values of $\eta_{10}$ obtained from analysis of two recombination channels provide confidence in validity of the results.

In order to verify the exceptionally high IQE of the BL band by an independent method, we measured dependences of the integrated PL intensities on excitation intensity at $200 \mathrm{~K}$. We used a simple relation between the excitation power density, $P_{\text {exc }}$, and the carrier generation rate: $G=1.6 \times 10^{23} P_{e x c}{ }^{7}$ Since the YL band intensity starts saturating at relatively low excitation intensity, ${ }^{1}$ only the exciton and BL bands were analyzed (Fig. 3). As expected, at low excitation intensity, the IQE for both bands is constant, i.e., their intensities increase linearly with the excitation intensity. At high excitation intensity, the $\mathrm{Zn}_{\mathrm{Ga}}$ acceptors become saturated with holes, and the BL band efficiency decreases. ${ }^{1}$ The dependence can be fitted with the model suggested in Ref. 9. More importantly, Eq. (4) can be used to fit the relative change in the exciton band IQE and to find $\eta_{10}$ with an independent method. The increase in $\eta_{2}$ at $G>10^{24} \mathrm{~cm}^{-3} \mathrm{~s}^{-1}$ is caused by the decrease in the BL band IQE. From the fit, we obtain $\eta_{10}=0.90 \pm 0.05$ for the BL band at $200 \mathrm{~K}$. Unfortunately, we were not able to determine $\eta_{10}$ more accurately with this method since the excitation power densities above $200 \mathrm{~W} / \mathrm{cm}^{2}$ were not available in our experiment. The advantage of this method is that $I_{i 0}^{P L}$ for the exciton emission is linearly proportional to $G$ in the entire range of the excitation intensities due to high concentration of free electrons in the studied sample.

Very high IQE of PL observed in this work does not contradict to the relatively high density of threading dislocations, of the order of $10^{9} \mathrm{~cm}^{-2}$, expected for GaN layers grown on sapphire by MOVPE. While the dislocations are commonly considered as the main source of nonradiative recombination in $\mathrm{GaN}$, the $\mathrm{Zn}$ acceptors with concentration above $10^{18} \mathrm{~cm}^{-3}$ and hole-capture cross-section of $\sim 10^{-13} \mathrm{~cm}^{2}$ (Ref. 1) can successfully compete with the nonradiative channel for capture of minority carriers (holes), provided that the doping does not create point nonradiative defects in large number.

In summary, we have fabricated high quality MOVPE GaN layers co-doped with silicon and zinc and used three approaches to determine the absolute IQE of PL, namely, (1) the characteristic temperature $T_{0}$ at which the thermal quenching of the BL band begins, (2) the value of the increase in intensity of PL bands caused by the quenching of the BL band, and (3) the value of the rise of the relative IQE for the exciton band caused by a decrease of the BL band efficiency at high excitation intensities. With these independent approaches, we have estimated the absolute IQE of the BL band as $\eta_{10} \approx 0.93 \pm 0.04$ at $T<250 \mathrm{~K}$. The methods used here are very accurate when the IQE approaches unity since the size of the effect (e.g., an increase of PL intensities for all PL bands caused by a quenching of a PL band with high IQE) becomes very large as compared to errors in modeling the $I_{i 0}^{P L}(T)$ or $I_{i 0}^{P L}\left(P_{e x c}\right)$ dependences. The nonradiative recombination efficiency is less than $10 \%$ at $250 \mathrm{~K}$ and $55 \%$ at room temperature.

The work was supported by the BMBF under the project "Ephquam."

${ }^{1}$ M. A. Reshchikov and H. Morkoç, J. Appl. Phys. 97, 061301 (2005).

${ }^{2}$ S. Nakamura, T. Mukai, and M. Senoh, Appl. Phys. Lett. 64, 1687 (1994).

${ }^{3}$ H. Y. Ryu, K. H. Ha, J. H. Chae, K. S. Kim, J. S. Son, O. H. Nam, Y. J. Park, and J. I. Shim, Appl. Phys. Lett. 89, 171106 (2006).

${ }^{4}$ Q. Dai, M. F. Schubert, M. H. Kim, J. K. Kim, E. F. Schubert, D. D. Koleske, M. H. Crawford, S. R. Lee, A. J. Fischer, G. Thaler, et al., Appl. Phys. Lett. 94, 111109 (2009).

${ }^{5}$ X. Ni, J. Lee, M. Wu, X. Li, R. Shimada, Ü. Özgür, A. A. Baski, H. Morkoç, T. Paskova, G. Mulholland, et al., Appl. Phys. Lett. 95, 101106 (2009).

${ }^{6}$ H. Yoshida, M. Kuwabara, Y. Yamashita, K. Uchiyama, and H. Kan, Appl. Phys. Lett. 96, 211122 (2010).

${ }^{7}$ M. A. Reshchikov, A. Kvasov, T. McMullen, M. F. Bishop, A. Usikov, V. Soukhoveev, and V. A. Dmitriev, Phys. Rev. B 84, 075212 (2011).

${ }^{8}$ M. A. Reshchikov and R. Y. Korotkov, Phys. Rev. B 64, 115205 (2001).

${ }^{9}$ M. A. Reshchikov, Appl. Phys. Lett. 88, 202104 (2006). 